\title{
Awareness of Emerging Novel Epigenetic Targeting Therapy in Acute Myeloid Leukemia
}

\author{
Nahla AM Hamed* \\ Hematology Department, Alexandria University, Egypt
}

Submission: June 11, 2018; Published: July 23, 2018

"Correspondence Address: Nahla AM Hamed, Professor of Hematology, Faculty of Medicine, Alexandria University, Hematology Department, Alexandria University, Egypt, Email: drhamedn@hotmail.com

\begin{abstract}
Many of the recurrently mutated genes in AML may affect epigenetic regulators of transcription. Epigenetic modifiers represent a major focus of interest and are highlighted as attractive targets for personalized therapy in AML. Some novel agents showed encouraging clinical activity in early phase trials while other agents (e.g., EZH2 inhibitors) are in early preclinical development and will likely be tested in AML trials in the near future. Many novel agents in development target niche areas for AML leukemogenesis including the epigenetic regulators BET proteins, DOT1L, and LSD1 which are suggested to have a role in MLL-r AML and possibly other subsets.

Abbreviations: AML: Acute myeloid leukemia; ITD: Internal Tandem Duplication; BM: Bone Marrow; IDH1: Isocitrate Dehydrogenase 1; TET2: Ten-eleven Translocation-2; ASXL1: Additional Sex Comb-like 1; RUNX1: Runt-Related Transcription Factor-1; DNMT3A: DNA methyltransferase 3A; HMAs: Hypomethylating Agents; DAC: Decitabine; AZA: Azacitidine; OS: Overall Survival; BET: Bromodomain and Extraterminal; MLL: Mixed Lineage Leukemic; LSD1(KDM1A): Lysine-specific Demethylase 1; CCI: Charlson Comorbidity Index; ELN: European Leukemia Net; HCTCI: Hematopoietic Cell Transplantation Comorbidity Index; MPN: Myeloproliferative Neoplasm; MRC: Myelodysplastic-related Change; SET: Suppressor of Variegation, Enhancer of Zeste, Trithorax; DOT1L: Disruptor of Telomeric Silencing 1-like; R/R: Relapsed/Refractory; MLL-r: MLLRearranged.
\end{abstract}

\section{Introduction}

Many of the newly identified recurrently mutated genes in AML frequently affect epigenetic regulators of transcription [1]. These epigenetic alterations disturb DNA promoter methylation or histone methylation/acetylation, resulting in pathogenic gene expression changes critical for enhanced stem cell renewal, impaired normal cell differentiation, and chemoresistance [1]. The most commonly mutated genes regulating the methylation and/or hydroxymethylation of cytosine bases in DNA include DNMT3A, WT1, IDH1/2, and TET2. All together are observed in $\sim 50 \%$ of AML cases. Mutations in genes altering transcription through histone modifications, such as ASXL1, EZH2, or KMT2A (formerly known as MLL) are frequently seen, in AML following antecedent MDS or prior chemotherapy [2]. Aberrant expression of the LSD1 (KDM1A) demethylase, overexpression of histone deacetylase 9 and the Polycomb group of proteins has been implicated in leukemia [3].

Loss-of-function aberrations in the SET domain of EZH2 have been found in $3 \%$ of primary AML, and $29 \%$ of secondary AML. All these perturbations lead to poor prognosis and diminished overall survival [3]. Mutations in these epigenetic modifiers are likely to promote clonal outgrowth but are insufficient to initiate leukemic transformation without subsequent mutational events [4]. Histone methylating/demethylating and acetylating/deacetylating enzymes "write" and "erase" these epigenetic marks, respectively [1] to re-establish physiological cell processes. Epigenetic enzymes represent attractive targets for cancer therapy [1]. Several novel epigenetic therapies have entered clinical trials or are in preclinical testing phases [5].

\section{Epigenetic Therapies}

\section{DNMT Inhibitors}

DNMT3A catalyzes de novo methylation of cytosine residues in DNA [2]. DNMT3A is recurrently mutated in $~ 20 \%$ of de novo AML [3], frequently in advanced age and in conjunction with NPM1, FLT3-ITD, and/or IDH1 mutations [4]. Several series have shown its correlation with chemotherapy resistance and disease relapse [4]. It is one of the 3 most common mutations in AML [3]. The most frequent DNMT3A mutation is the R882 missense mutation which prevents methyl transferase activity and DNA binding [4]. Decitabine (DAC) and 5-azacitidine (AZA) are pyrimidine analogs acting as DNMT inhibitors, leading to global hypomethylation of cytosine residues, at cytosine guanine dinucleotide-rich gene promoters and at distal enhancers critical for gene expression regulation [2]. These drugs are backbone for combination strategies in older patients 


\section{Cancer Therapy \& Oncology International Journal}

with epigenetic mutations and those at high risk for treatment failure and/or unacceptable toxicity with standard intensive approaches due to adverse genetics or comorbidity [2]. An extended 10-day schedule of DAC in elderly patients ( $>60$ years), is associated with a higher complete response rate of $47 \%$ (1). They are very tolerable and often effective therapy of AML [2]. The mechanisms responsible for their antileukemia activity are poorly understood [4].

Factors that favor use of epigenetic therapy over chemotherapy in poor-risk AML patients

Final decisions are highly individualized, in view of balanced benefit/risk [2]. Age $\geq 80$ yr, ECOG PS $\geq 2$, high CCI, HCT-CI ( $\geq 3)$, lower $\mathrm{WBC}<15,000 / \mathrm{cmm}$, secondary AML (post-MDS, post MPN), MRC-AML by WHO classification, unfavorable genetics: monosomy 5 or 7, del (5q), complex, monosomal karyotype, TP53 gene mutation, no FLT3-ITD, epigenetic gene mutations (TET2, DNMTA) favor the use of epigenetic therapy [2].

\section{Genetic predictors of HMA response}

Mutations in TET2 or DNMT3A, adverse-risk cytogenetics and/or TP53 gene mutations has been suggested to predict higher response rates without survival benefit to DAC treatment in AML patients compared with other patients [2]. Dual presence of DNMT3A and NPM1 mutations may correlate with responses to HMAs in frontline and combined frontline/ relapsed/refractory settings, whereas DNMT3A mutation alone was associated with a higher response in frontline setting [1]. No confirmed relationship between the presence or absence of epigenetic mutations, degree of demethylation (pre- or postHMA treatment), and patient outcome [4].

Selected combination Strategieswith Hypomethylating Agents

Single hypomethylating agents are not superior to intensive cytotoxic approaches [2]. The combinations of epigenetic-acting agents (HMAs and histone deacetylase inhibitors) failed to have their expected impact [6]. Sorafinib may be added to HMAs in patients with FLT-ITD mutation. Azacytidine followed by donor lymphocyte infusions was suggested as treatment option for relapsed AML after allogeneic HCT [7]. HMAs in combination with the BH3-mimetic venetoclax have promising efficacy in unfit elderly AML patients in a phase $1 \mathrm{~b} / 2$ study. Addition of ATRA to DAC schedule in unfit AML patients $\geq 60$ years in DECIDER study improved survival, despite a non-significant improvement in response. ATRA has been proposed to downregulate BCL-2 in AML blasts/progenitors [1]. Combining HMA with checkpoint inhibitors therapy has generated promising early clinical results [1]. Additional work is required to determine whether molecularly defined secondary AML patients [2] or cytarabine refractory AML patients with poor-risk molecular or cytogenetic features may benefit from epigenetic therapy [4]. It is possible that the activity of HMAs might be missed if they are studied only in patients with active AML rather than in remission [6].
Novel epigenetic modulators in clinical development in AML [8]
i. New DNMT inhibitors (SGI-110)
ii. HDAC inhibitors (panobinostat, pracinostat)
iii. IDH1 and IDH2 inhibitors
iv. DOT1L (KMT4) inhibitors (pinometostat)
v. BET-bromodomain inhibitors
a. LSD1 (KDM1A) inhibitors (GSK2879552)
b. EZH2 (KMT6) inhibitors (tazemetostat)
c. PRMT5 inhibitors (GSK3326595)
d. BRD4 inhibitors (MK-8628)

\section{New DNMT (Novel HMAs)}

Oral formulation of AZA (CC-486) and a second generation HMA, guadecitabine (SGI-110) are under investigation [6]. Guadecitabine is a dinucleotide of decitabine and deoxyguanosine in phase 3 development. It increases the in vivo exposure of decitabine by protecting it from inactivation by cytidine deaminase [8]. There are limited data for clinical activity of CC-486 in AML. A phase 1/2 trial of CC-486 as maintenance therapy after allogeneic transplant for AML has been completed, and a phase 3 study of this drug as maintenance therapy after completion of intensive non transplant chemotherapy is ongoing (identifier NCT01757535). It might emerge as a drug of choice for combination regimens in AML in the future [2].

\section{IDH1 and IDH2 Inhibitors}

IDH1-R132, IDH2-R140, and IDH2-R172 occur in 20\% of $\mathrm{AML}$, and are more frequent in intermediate risk AML, advanced age, and concurrent with NPM1 mutations. IDH1/IDH2 mutations reduces $\alpha$-KG into the oncometabolite 2-hydroxyglutarate, which then competitively inhibits $\alpha$-KG-dependent reactions [4]. Early trials with IDH1 and IDH2 inhibitors show encouraging early results [4] with durable responses [8]. Targeted inhibitors of IDH2 (enasidenib, formerly AG221), IDH1 (AG120, IDH305, and FT2102), or pan-IDH1/IDH2 (AG881) are in clinical development. Additional ongoing clinical trials include $7+3+$ enasidenib or AG120 (\#NCT02632708) and azacitidine + enasidenib or AG120 (\#NCT02677922), as frontline therapy for newly diagnosed AML patients, and a randomized phase 3 "IDHENTIFY" trial of enasidenib for R/R AML with IDH2 mutation (\#NCT02577406) [4].

\section{HDAC Inhibitors (HDACi)}

The addition of panobinostat to azacitidine improved response rate, but not survival among AML patients in a randomized phase $2 \mathrm{~b}$ study. Another phase 2 study produced a more promising CR rate of $42 \%$ with pracinostat in combination with azacitidine [1]. 


\section{Cancer Therapy \& Oncology International Journal}

\section{D0T1L Inhibitors}

Rearrangements of the MLL gene at the 11q23 chromosome locus are present in $5-10 \%$ of AML cases and portend poor prognosis. Most of the MLL fusion partners bind to DOT1L, which is postulated to be the oncogenic driver of MLL-r AML via its histone methyl transferase activity. Pinometostat (EPZ-5676) is a DOT1L inhibitor with an acceptable safety profile. An ORR of $12.2 \%$ in adult $\mathrm{R} / \mathrm{R}$ acute leukemias patients and no ORR in $\mathrm{R} / \mathrm{R}$ MLL-r children acute leukemia was demonstrated in early phase 1 trials. Target inhibition was shown in both studies. Pinometostat combinations with other anti-leukemic agents should be explored in next steps. Preclinical work suggests that these agents may play a large role in NPM1/ FLT3-ITD-mutated leukemias [5].

\section{LSD1 Inhibitors}

LSD1 is a histone demethylase expressed in leukemic cells and regulates the differentiation block in AML [5]. LSD1 inhibitors have shown antileukemic activity in vitro and striking hematopoietic toxicity in preclinical models [9]. GSK2879552 and ORY-1001 have entered early-phase trials for R/R acute leukemia patients (NCT02177812, EudraCT number 2013002447-29) [5].

\section{BET inhibitors}

BET proteins bind acetylated histone tails and recruit the transcriptional machinery to the promoter regions of genes [5]. BET inhibitors include OXT015, CPI-0610, TEN-010, GSK525762, and INCB054329 [4]. These inhibitors have demonstrated formidable preclinical activity, and several are currently under evaluation in phase $1 \mathrm{R} / \mathrm{R}$ AML studies (\#NCT02158858, \#NCT02308761, \#NCT01943851, and \#NCT02308761) [4], both as single-agent and in combination with standard therapies and other novel agents [5]. Common AEs were diarrhea and hyperbilirubinemia. No biomarkers to predict response have been identified [5]. Other examples are target of KMT2A (MLL)rearranged leukemias or of BRD4, a member of the BET family of bromodomain epigenetic readers [8], and an adapter for chromatin modification [1].

\section{Conclusion}

Evaluation of gene-gene interactions, not only single mutations, will be essential to better identify treatment strategies. The optimal use of these targeted agents requires studies performed earlier in the disease course most likely in combination with other antileukemic therapy. Utilization of accurate biomarkers of clinical response and/or genetic predictors of response as well as identifying markers of resistance might provide improved prognostication and change AML management in the near future.

\section{References}

1. Wei A (2018) Epigenetic treatment and beyond in acute myeloid leukemia. Educational Updates in Hematology Book 2(S2): 19-22.

2. Dombret H, Itzykson R (2017) How and when to decide between epigenetic therapy and chemotherapy in patients with AML. ASH Education Book 2017(1): 45-53.

3. Sbirkov Y, Kwok C, Bhamra A, Thompson AJ, Gil V, et al. (2017) Semiquantitative mass spectrometry in AML cells identifies new nongenomic targets of the EZH2 methyltransferase. Int J Mol Sci 18(7): 1440 .

4. DiNardo CD, Cortes JE (2016) Mutations in AML: prognostic and therapeutic implications. ASH Education Book 2016(1): 348-355.

5. Saygin C, Carraway HE (2017) Emerging therapies for acute myeloid Leukemia. Journal of Hematology \& Oncology 10(1): 93.

6. Tefferi A, Estey EH (2016) Acute myeloid leukemia: 2016 Update on risk-stratification and management. J Hematol Oncol 91(8): 824-846.

7. NCCN.org (2017) NCCN Clinical Practice Guidelines in Oncology (NCCN Guideline Version 1.2017- February 24, 2017.s) Acute myeloid leukemia, Washington, USA.

8. Dohner H, Estey E, Grimwade D, Amadori S, Appelbaum FR, et al (2017) Diagnosis and management of AML in adults: 2017 ELN recommendations from an international expert panel. Blood 129(4): 424-447.

9. Moarii M, Papaemmanuil E (2017) Classification and risk assessment in AML: integrating cytogenetics and molecular profiling. Hematology 2017(1): 37-44

\begin{tabular}{|l|}
\hline \multicolumn{1}{|c|}{ Your next submission with Juniper Publishers } \\
will reach you the below assets \\
- Quality Editorial service \\
- Swift Peer Review \\
- Reprints availability \\
- E-prints Service \\
- Manuscript Podcast for convenient understanding \\
- Global attainment for your research \\
- Manuscript accessibility in different formats \\
( Pdf, E-pub, Full Text, Audio) \\
- Unceasing customer service \\
Track the below URL for one-step submission \\
https://juniperpublishers.com/online-submission.php \\
\hline
\end{tabular}

